\title{
Automated Health Maintenance Reminders: Tools Do Not Make a System
}

\author{
Paul S. Frame, MD
}

Computer-based reminders to prompt physicians to implement preventive and other services have been available since the late 1970s, when, they were pioneered by McDonald and colleagues ${ }^{1}$ at the Reigenstrief Clinic. These tools offer great promise for improved patient care and increased practice efficiency. Studies have reported modest improvements in delivery of preventive services using automated reminders. ${ }^{2}$ Their use, however, is not widespread or always successful.

In this issue of the journal, Schellhase et $\mathrm{al}^{3}$ report results of a survey of provider reactions to automated reminders for preventive services delivered in the context of an electronic medical record. Their results are informative for their honesty and depressing in their conclusions. Seventy-five percent of clinicians reported not paying attention to the flashing reminder icon, and only $20 \%$ reported regularly reviewing health maintenance needs of the patient before the clinical encounter.

I believe the most important reason for the negative results reported in this survey is that the practice described has a tool to remind providers of needed preventive services but does not have a system to ensure optimal delivery of preventive services. A system is a process for achieving a desired goal. Tools are usually an important component of systems but are not a substitute for a system.

Necessary components of a system for delivering preventive services include: $^{4}$

1. A written practice health maintenance protocol

2. Specific delegation of responsibility for doing preventive procedures

Submitted 10 February 2003.

From Tri-County Family Medicine, Cohocton, and the University of Rochester School of Medicine and Dentistry, Rochester, NY. Address reprint requests to Paul S. Frame, MD, Tri-County Family Medicine, 25 Park Ave, Cohocton, NY 14826.
3. Involvement of patients

4. A system of periodic audits to evaluate whether goals are being met

5. Provider (and possibly patient) reinforcements for compliance with the health maintenance protocol

In a recent meta-analysis of interventions that increase the use of preventive services, Stone et $\mathrm{al}^{5}$ found practice organizational changes, including staffing and clinical procedures, to be the most effective. Involving patients using financial incentives or reminders was also effective. Provider reminders alone were less effective.

The practice described by Schellhase et al in this issue of the journal has parts of a system for implementing preventive services. They presumably have a health maintenance protocol based on selected recommendations of the US Preventive Services Task Force. They have an electronic record (EMR) that prompts providers when procedures are due. This EMR is capable of facilitating audits of provider health maintenance compliance and sending patient health maintenance reminders.

The practice now needs to designate a person or group to have ownership of the development and maintenance of the prevention system. This entity is responsible for ensuring goals are achieved.

Next, the process for the system needs to be developed. Are providers responsible for preventive services, or should preventive services be delegated to other personnel? If providers are responsible for prevention, are they expected to address it at every visit or just health maintenance visits? If only at health maintenance visits, are all patients being prompted to schedule such visits?

A baseline audit should be conducted to determine current provider compliance with the health maintenance protocol. The EMR should allow this audit to be done electronically. Periodic audits should be scheduled so that progress toward meet- 
ing the compliance goals can be measured. If progress is not occurring, the system might need to be modified. Finally, the practice should determine what provider (and patient) incentives will be used to encourage compliance. Provider incentives can consist of peer cajoling during review of audit results or can be more direct, such as financial rewards for good performance.

Developing and maintaining a system to deliver preventive services requires effort and resources. It will not occur spontaneously. The results will justify the effort in terms of quality of care and provider and patient satisfaction.

\section{References}

1. McDonald CJ, Hui SL, Smith DM, et al. Reminders to physicians from an introspective com- puter medical record. A two-year randomized trial. Ann Intern Med 1984;100:130-8.

2. Frame PS, Zimmer JG, Werth PL, Hall JW, Eberly SW. Computer-based vs manual health maintenance tracking. A controlled trial. Arch Fam Med 1994;3: 581-8.

3. Schellhase KG, Koepsell TD, Norris TE. Providers' reactions to an automated health maintenance reminder system incorporated into the patient's electronic medical record. J Am Board Fam Pract 2003; $16: 312-7$.

4. Frame PS. Implementing clinical preventive medicine: time to fish or cut bait. J Am Board Fam Pract 2000;13:84-5.

5. Stone EG, Morton SC, Hulscher ME, et al. Interventions that increase use of adult immunization and cancer screening services: a meta-analysis. Ann Intern Med 2002;136:641-51. 